\title{
Seeing colors in real scenes
}

\author{
Sérgio M. C. Nascimento \\ Centre of Physics, Campus de Gualtar, University of Minho, 4710-057, Braga, Portugal
}

\begin{abstract}
Color perception in real conditions is determined by the spectral and spatial properties of objects and illumination. These properties are best evaluated by spectral imaging, a technique that records the reflecting spectral profile for each point of the scene. Using this technique on a set of natural scenes it was found that the color gamut expressed in the CIELAB color space is much smaller than the theoretical limits defined for the object colors. Moreover, the colors more frequent are those around the white point and their frequency of occurrence can be well described by a power law. Spatial variations of the spectral composition of the illumination across natural scenes were also quantified by placing small reflecting spheres in different locations of the scenes. The extent of these variations across scenes was found to be large and of the same order of magnitude as the variations of daylight along the day. These findings show that colors in nature are considerable constrained and that constancy mechanisms must be efficient over a wide range of stimuli variations to compensate for large natural variations of illumination.
\end{abstract}

Keywords: color vision, spectral imaging, natural colors, color rendering, color constancy

\section{INTRODUCTION}

The evolution of the visual system was constrained by the properties of the visual environment. Understanding how vision encodes these properties implies their precise quantification. For color vision the relevant data concerns spectral information and the way it is related with the spatial structure of natural scenes. What is the gamut of natural colors and how does it compare with the theoretical maximum? What are the colors that occur more often in nature? These and other related questions can be studied by empirical measurements in natural scenes. Spectral imaging is a technique that records spectral information for different points of a scene and, therefore, is the best technique to characterize natural stimuli to address these questions.

Spectral imaging requires an imaging device, usually a CCD camera, coupled with a spectral filter, usually a tunable filter ${ }^{1}$. Figure 1 shows the spectral imaging system used to obtain the data described in this paper. The system was composed with a low-noise Peltier-cooled digital camera capable of a spatial resolution of $1344 \times 1024$ pixels (Hamamatsu, model C4742-95-12ER, Hamamatsu Photonics K. K., Japan), and a fast tunable liquid-crystal filter (Varispec, model VS-VIS2-10-HC-35-SQ, Cambridge Research \& Instrumentation, Inc., Massachusetts) mounted in from of a lens. Each image was acquired from $400-720 \mathrm{~nm}$ in $10 \mathrm{~nm}$ steps. The spatial resolution of the system was at least as good as the human eye at the same distance. The spectral radiance for each pixel was estimated using the radiance acquired from a known gray reference presented in the scene at the time of acquisition, measured with a telespectroradiometer (SpectraColorimeter, PR-650, PhotoResearch Inc., Chatsworth, CA), calibrated in the National Physical Laboratory. The system was used to acquired spectral data from natural scenes classified in rural and urban (for more details on the system see $^{2}$ ).

The theoretical limits for the object colors in nature are the optimal colors which enclose the object-color solid representing all colors arising only by reflection or transmittion ${ }^{3}$. Colors arising from other processes, e.g. highlights, fluorescence, Rayleigh or Mie scattering, are not considered as object colors and may be represented outside the objectcolor solid. The theory underlining the spectral properties of optimal colors was developed early in the 20th century ${ }^{4}$ and the corresponding loci were computed later by David L. MacAdam ${ }^{5}$ to obtain the MacAdam limits. Figure 2 shows the representation of the object-color solid for the CIE standard illuminant D65. 

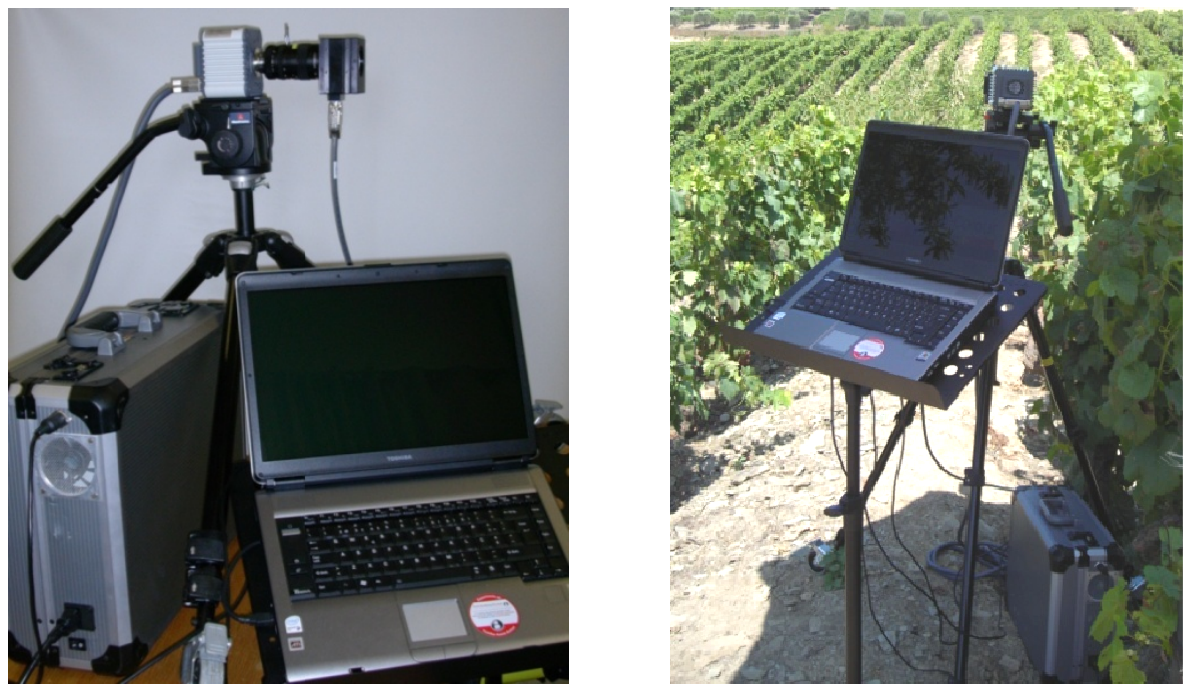

Figure 1.Spectral imaging system used to obtain the spectral data for the computations described in this paper.

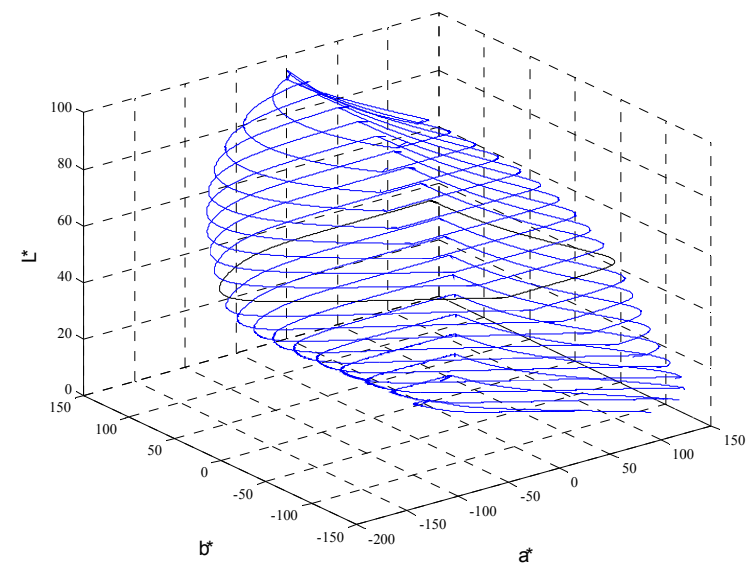

Figure 2. Representation of the object-color solid for the CIE standard illuminant D65. Lines represent the MacAdam limits.

\section{GAMUT OF NATURAL COLORS}

To derive the gamut of natural colors spectral imaging data for a set of 50 natural scenes were analyzed. Some of the scenes of this set are represented in Figure 3. The spectral radiance of each individual pixel was converted into tristimulus values, assuming the CIE 1931 standard colorimetric observer, and then converted into CIELAB color coordinates. Figure 3 shows the color gamut of the 50 scenes represented in the CIELAB color space. For simplicity only the data for $\mathrm{L}^{*}=50$ is represented. Also represented is the contour of the optimal colors for the CIE standard illuminant $\mathrm{C}$ and the color measured by Pointer ${ }^{6}$ which includes large sets of colored samples containing colors of paints, flowers, plastics, inks, among others. The gamut of the natural scenes is much smaller than that defined by the MacAdam limits and is close to the limits defined by the colors measured by Pointer. 
The number of discernible colors that can be perceived has been a matter of analysis in the history of color science. It was initially estimated using chromatic discrimination data ${ }^{7}$ but recent estimates are based on representations in uniform color spaces ${ }^{8-10}$. One estimate for the complete object-color volume is close to 2 million ${ }^{9}$. Here the number of perceived colors was estimated as follows. Two topological packing methodologies were applied. In the cubic packing method, the CIELAB color volume for each scene was segmented in unitary cubes and the number of non-empty cubes counted ${ }^{9}$. This methodology assumes that all colors represented inside the same cube are cannot be discriminated and naturally underestimates the number of colors as the maximum distance between 2 points packed into the same cube can be larger than 1. In the spherical packing method, the color volume was segmented in spheres instead. This methodology produces a larger estimate of the number of colors than that based in a cubic packing and may be a more realistic topology for estimating the number of discernible colors perceived by humans.
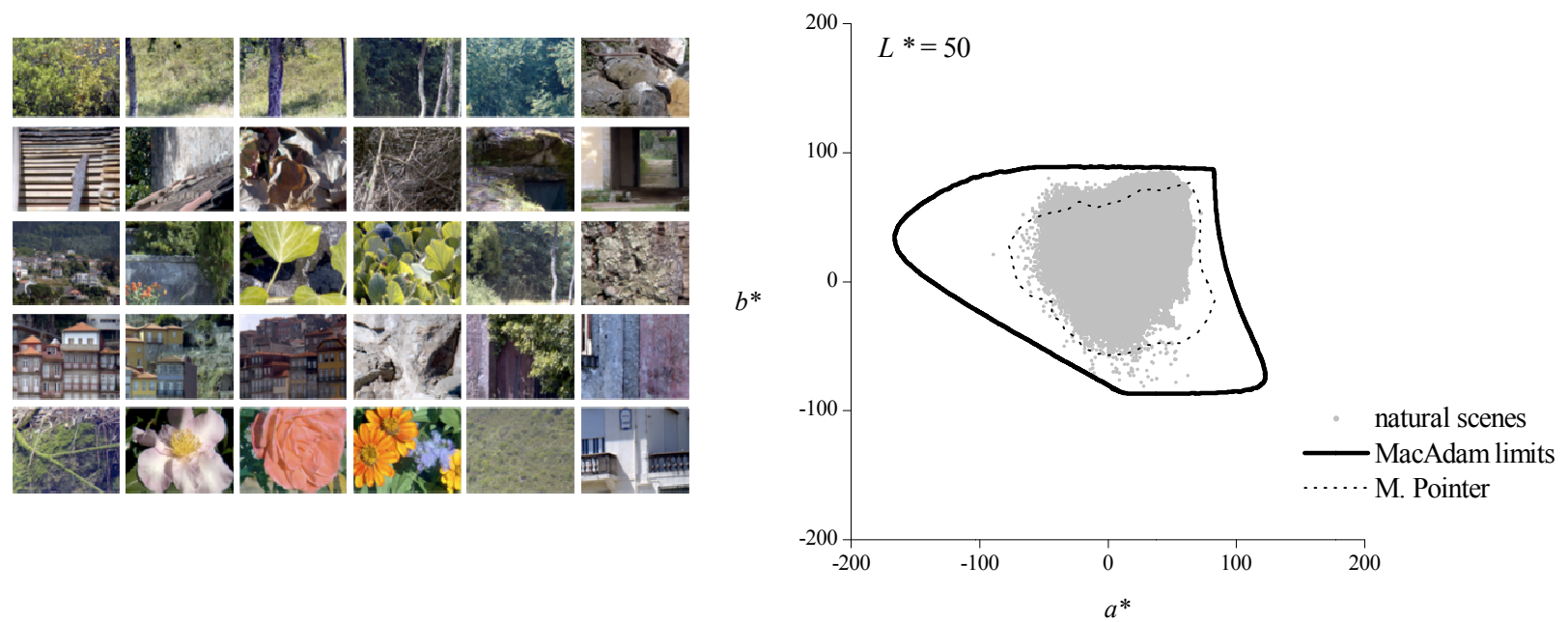

Figure 3. Left: colored pictures of some scenes of the set of 50. Right: the color gamut of the 50 scenes analyzed represented in the CIELAB color space. For simplicity only the data for $\mathrm{L}^{*}=50$ is represented. Also represented is the contour of the MacAdam limits for the CIE standard illuminant $\mathrm{C}$ and the colors measured by Pointer (see text for more details).

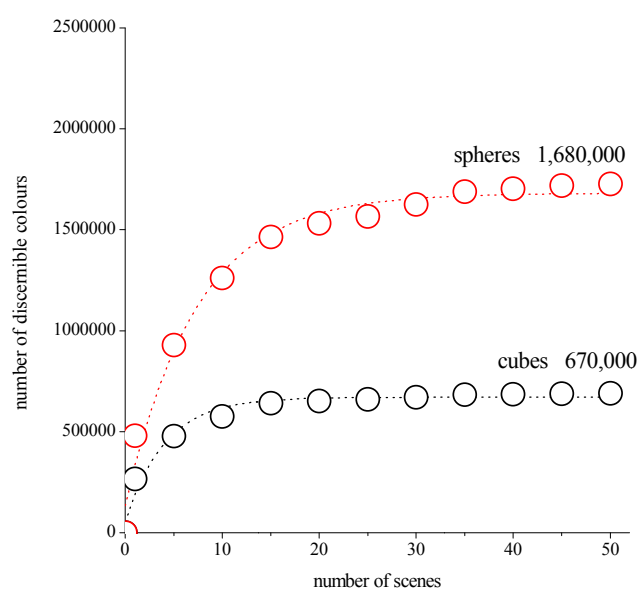

Figure 4. Number of discernible colors expressed as a function of the number of natural scenes analyzed. Data obtained by segmenting the color space in cubes or spheres. 
Figure 4 shows the number of discernible colors obtained with the two methodologies expressed as a function of the number of scenes of the database analyzed. The smooth curve represents an exponential fit to the data and the numbers on the graphs indicate the asymptotic values. For the methodology based on cubes the number obtained was about 670,000 . This value is less than half that obtained for the complete object color volume ${ }^{9}$.

\section{DISTRIBUTION OF NATURAL COLORS}

The distribution of natural colors was analyzed in the coordinate $\mathrm{L}^{*}$ and in CIELAB the $\left(\mathrm{a}^{*}, \mathrm{~b}^{*}\right)$ plane. Figure 5 represents these two distributions. The mean for $\mathrm{L}^{*}$ the distribution is 40 and for the distribution in the plane $\mathrm{a}^{*} \mathrm{~b}^{*}$ was $(0,12)$, that is very close to the white point.
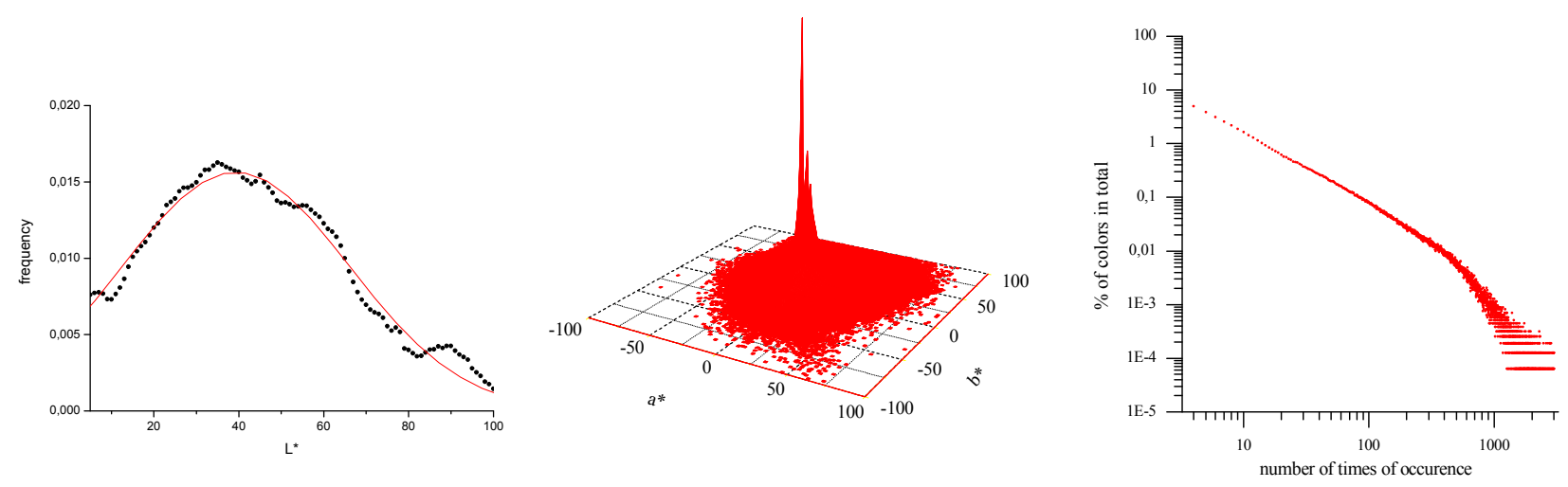

Figure 5. Distribution of colors in $\mathrm{L}^{*}$ (left) and in the CIELAB $\left(\mathrm{a}^{*}, \mathrm{~b}^{*}\right)$ plane (centre). On the right is represented the fraction of discernible colors as a function of the number of times of occurrence in the natural scenes.

By counting how often a particular color appears it is possible to estimate a frequency of occurrence for each color. Figure 5 shows on the right the fraction of discernible colors expressed as a function of the number of times of occurrence in the natural scenes. These data show that most colors are rare colors, that is, they can be seen only a very limited number of times. The form of the variation shows that it can be described by a power function.

\section{SPATIAL VARIATIONS OF ILLUMINATION}

During the day the colour of daylight changes from bluish to reddish. These chromatic changes are large and correspond to correlated colour temperature (CCT) changes within the approximate range of $40,000 \mathrm{~K}-4000 \mathrm{~K}^{11,12}$. The spectral distribution of the illumination also varies in space due to phenomena like occlusions and mutual reflections ${ }^{13,14}$. Yet, the visual system does not seem to perceive these variations ${ }^{15}$ due to mechanisms of compensation. To characterize these variations across natural scenes spectral imaging data was obtained from the scenes of the database. Before image acquisition a set of grey spheres was positioned in different locations of the scenes as illustrated in Figure 6 . These spheres were painted with a matte grey paint with known spectral reflectance. This methodology allows the derivation of the angular and spatial variations in each location. Figure 7 shows, as an example, the colors represented in CIE (x, y) diagram of the illuminant in the different locations of the scene represented. The analysis of the data from several scenes 
shows that the extent of these chromatic variations of the illuminant across scenes is of the same order as the variations along the day ${ }^{16}$.
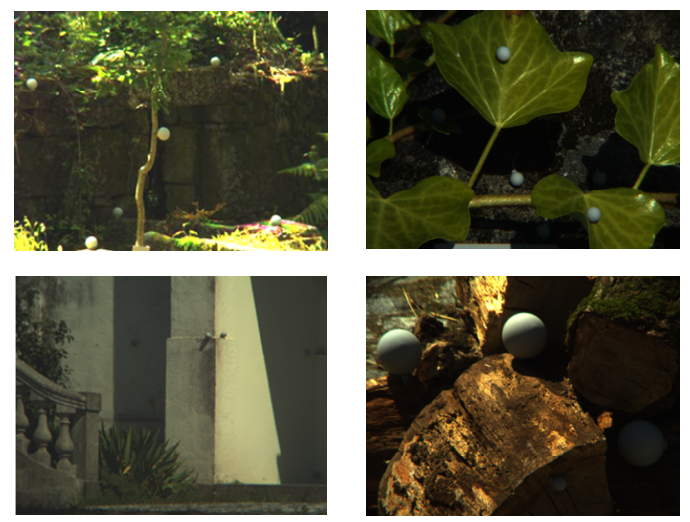

Figure 6. Examples of several scenes containing grey spheres to derive the spatial variation of the illumination.
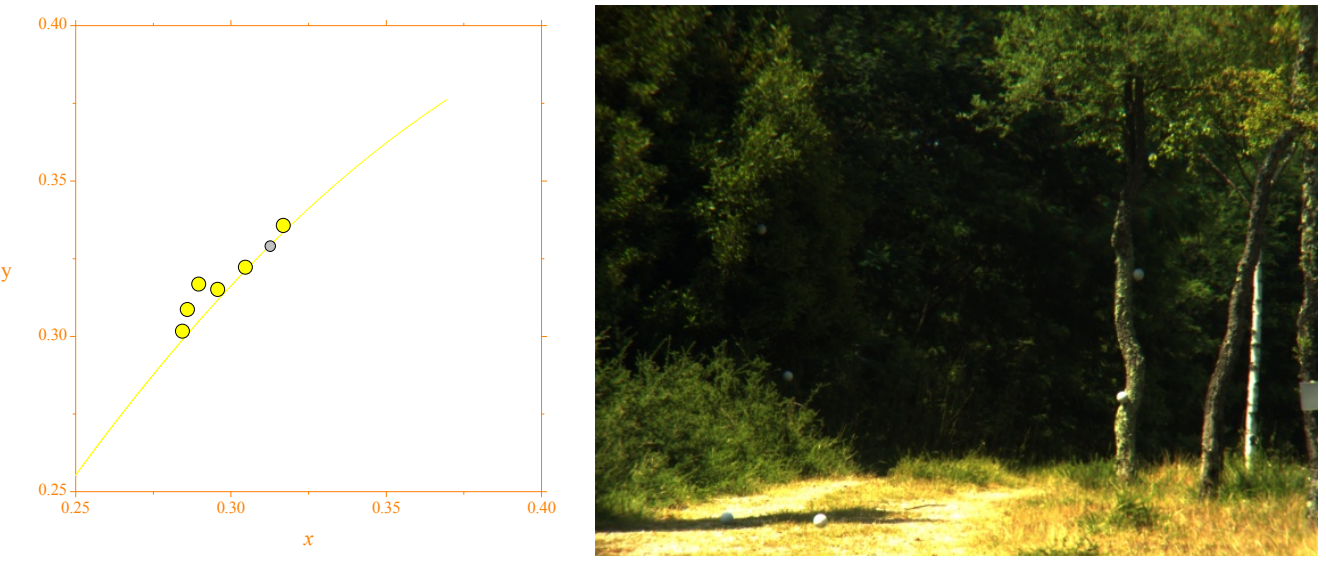

Figure 7. The colors of illumination represented in CIE $(x, y)$ measured for the different spheres located in the scenes to the right.

\section{SUMMARY AND CONCLUSIONS}

The data described here concerning the gamut of natural colors show that a considerable part of the theoretical object-color volume is not filled with natural colors and therefore natural stimuli seem less demanding to the visual system than might have been anticipated from the analysis of the theoretical limits. To what extent is the database analyzed representative of the colors of the natural world? The number of scenes is limited and they were acquired all in one region thus it is expected to contain some bias. However, the graph shown in Figure 4 suggests that as the number of scenes analyzed increases an asymptotic behavior seems to be obtained. This may mean that the scenes of the database represent a considerable part of natural colors. The data regarding the frequency of occurrence of colors show that unsaturated colors are the colors more frequent and that more saturated colors are rare. Also, it was found that most colors in natural scenes are rare a fact that can be described by a power law. Color gamut and frequency of occurrence suggest that different regions of the theoretical object-color space have different importance and meaning to the visual system which may imply that color vision has also equivalent non-uniform performance in some tasks. 


\section{ACKNOWLEDGMENTS}

This work was supported by the Centro de Física of Minho University, Braga, Portugal, and by the Fundação para a Ciência e a Tecnologia (grant PTDC/EEA-EEL/098572/2008). I am grateful to João M. M. Linhares, David H. Foster and Kinjiro Amano for the data regarding natural scenes.

\section{REFERENCES}

[1] Gat, N. "Imaging spectroscopy using tunable filters: a review," In: Szu HH, Vetterli M, Campbell WJ, Buss JR, eds.Vol 4056, 50-64 (2000).

[2] Foster, D. H, Nascimento, S. M. C., Amano K., "Information limits on neural identification of colored surfaces in natural scenes," Visual Neurosci., 331-336 (2004).

[3] Wyszecki, G., Stiles, W. S., [Color Science: Concepts and Methods, Quantitative Data and Formulae], New York: John Wiley \& Sons (1982).

[4] Schrodinger, E., "Theorie der pigmente von grosster leuchtkraft," Annalen Physik., 62:603-622 (1920).

[5] Macadam, D. L., "Maximum visual efficiency of colored materials," J. Opt. Soc. Am., 25:316-367 (1935).

[6] Pointer, M. R., "The gamut of real surface colours," Color Res. and Appl., 5:145-155 (1980).

[7] MacAdam, D. L., "Note on the number of distinct chromaticities," J. Opt. Soc. Am. 37, 308-309 (1947).

[8] Martinez-Verdu, F., Perales, E., Chorro, E., de Fez, D., Viqueira, V., and Gilabert, .E, "Computation and visualization of the MacAdam limits for any lightness, hue angle, and light source," J. Opt. Soc. Am. A 24, 1501-1515 (2007).

[9] Pointer, M. R., Attridge, G. G., "The number of discernible colours," Color Res. and Appl., 23:52-54 (1998).

[10] Linhares, J. M. M., Pinto, P. D., Nascimento, S. M., "The number and distribution of descernible colors in natural scenes, " J. Opt. Soc. Am. A 25, 2918-2924. (2008).

[11] Judd, D. B., MacAdam, D. L., Wyszecki, G., "Spectral distribution of typical daylight as a function of correlated color temperature," J. Opt. Soc. Am., 54:1031-1040 (1964).

[12] Hernandez-Andres, J., Romero, J., Lee, R.L., "Colorimetric and spectroradiometric characteristics of narrow-fieldof-view clear skylight in Granada, Spain" J. Opt. Soc. Am. A 18, 412-420 (2001).

[13] Endler, J. A. "The color of light in forests and its implications," Ecol. Monogr. 63, 1-27 (1993).

[14] Chiao, C. C., Cronin, T. W., Osorio, D., "Color signals in natural scenes: characteristics of reflectance spectra and effects of natural illuminants," J. Opt. Soc. Am. A. 17, 218-224 (2000).

[15] Almeida, V. M. N. D., "Perception of illuminant colour changes across real scenes" Perception 38, 1109-1117 (2009).

[16] Nascimento, S. M. C., Foster, D. H. , Amano, K., "Spatial and directional distributions of surface illumination in natural scenes analyzed by hyperspectral imaging," Invest. Ophth. Vis. Sci., 46 (2005) 